\title{
Design and Implementation of Campus Sharing Umbrella App
}

Lei Yu, Jiakun Huang, Kunyan Chen, Wangfei Zhou, Jing Wang, Hengyu Zhang*

Heilongjiang Bayi Agricultural University, Daqing163319 China

\author{
*Corresponding Author
}

Hengyu Zhang

\section{Article History}

Received: 10.02 .2020

Accepted: 18.02 .2020

Published: 29.02 .2020

\begin{abstract}
With the increasing popularity of the sharing economy, sharing umbrellas have gradually entered the public eye. In response to the complex climatic conditions in Daqing, design a "rain and shine" campus shared umbrella APP for students and staff. This program is based on the current sharing economy service form and aims to create a harmonious campus with integrity idea. Students and staff can find the umbrella borrowing points set on campus through mobile phone GPS, and use WeChat QR codes to pay for unlocking, borrow umbrellas, and solve their needs for umbrellas in various weather conditions to improve the happiness of campus life.
\end{abstract}

Keywords: Shared umbrella; QR code; GPRS technology.

\section{OVERVIEW}

In the era of "sharing economy", "sharing umbrella" is also one of the trends. At present, the "sharing umbrella" has not entered the right track operation, so we narrowed down the scope, starting from campus, considering school teachers and students, relying on school management methods, focusing on service, supplemented by profit, and grasped both aspects to create sharing a healthy development environment for sunny umbrellas. According to the information, the current problems are mainly the damage to the umbrella and the inconvenience of borrowing. Aiming at the problem of damage, the use of umbrellas must have a life span, and there are also malicious damage, possession, and random stacking, which are the main problems that cause umbrella damage. We take schools as the place and rely on school management methods. Because the scope of use is relatively small, we can "seat the seats right", which greatly reduces the phenomenon of uncivilized use. In view of the inconvenience of borrowing and repaying, because we mainly solve it from the time of use, increasing the time of use can alleviate the urgency of the teacher and student's use, and can use today to repay tomorrow. The "shared" service is mainly implemented through the use points of the station piles, which will cause the problem of the difference in the loan and return ratio. The common phenomenon is that most of them have no location when returning the umbrella. Location, for which we increase the use time can also alleviate the problem of the difference between the loan and return ratio to a certain extent.

\section{SOFTWARE REQUIREMENTS ANALYSIS AND SYSTEM DESIGN Requirements analysis}

The purpose of the "Along with Sunshine and Rain" campus shared umbrella app is to meet the needs of all teachers and students for umbrellas in various weather conditions, and to achieve the on-demand availability of rain umbrellas at the nearest distance, and return as needed. With the "Along with Sunshine and Rain" campus shared umbrella app, teachers and students can provide functions such as finding, borrowing, and returning umbrellas when they need to use the umbrella.

Copyright @ 2020: This is an open-access article distributed under the terms of the Creative Commons Attribution license which permits unrestricted use, distribution, and reproduction in any medium for non commercial use (NonCommercial, or CC-BY-NC) provided the original author and source are credited.

(C) South Asian Research Publication, Bangladesh $\quad$ Journal Homepage: www.sarpublication.com/sarjaf

"Sunny and rainy companion" campus Shared umbrella system and realization

XC2019016 


\section{System design}

The shared umbrella APP system is divided into three layers as a whole, the perception layer, the network layer and the application layer. The function of the APP includes 3 modules, GPS positioning module, umbrella module, and transaction module.

\section{GPS Positioning module}

This module can be divided into campus positioning and real-time query. The first step to enter the software is the GPS positioning interface. Through the GPS positioning interface, the user locates his location, finds the umbrella borrowing point, and selects an umbrella at the borrowing point to scan the code for borrowing, and uses the reference in this module. The map obtains latitude and longitude, and accurately limits the range of returning the umbrella to the point of borrowing and returning, ensuring that users are facing the teachers and students at school, but does not limit whether the range of using the umbrella is on campus, highlighting the convenience and practicality of the APP.

\section{Borrowing an umbrella module}

The module is divided into: real-name authentication, query, payment of deposit, and QR code payment. Because this program is only for teachers and students in the school, strict real-name authentication is required. Umbrella borrowers must bind their student ID and school.

\section{Trading module}

This module is divided into two parts: buying umbrellas and selling umbrellas. Due to the changing weather or inaccurate weather forecasts, teachers and students who need umbrellas occasionally appear and intend to buy umbrellas, and some students have no intentions because they change new umbrellas or are near graduation. Taking away the lowpriced processing and selling umbrellas can well ensure that resources are not wasted and recycled, and the sharing economy is combined with second-hand transactions, highlighting the program's environmental protection, sharing, convenience and mutual benefit.

\section{Share Umbrella App Design}

To meet the storage needs of users and shared umbrella information in the development of Android, the "Along with Sunshine and Rain" campus shared umbrella app chose to use the SQLite database to store data. The main database tables established are shown in Tables 1 .

Table-1: Sale Umbrella the main structure of the table

( Used to store user selling umbrella information )

\begin{tabular}{|l|l|l|}
\hline \multicolumn{3}{|c|}{ the main structure of the table } \\
\hline 1 & user_id & user id, Unique primary key \\
\hline 2 & price & User-defined umbrella prices \\
\hline 3 & content & $\begin{array}{l}\text { User described umbrella } \\
\text { information }\end{array}$ \\
\hline 4 & phone_number & User Contact \\
\hline 5 & Is_sale & Has been sold \\
\hline
\end{tabular}

To facilitate the operation of the database, the Green-Dao database management framework was introduced on the basis of SQLite. According to the configuration information of Green Dao, integrate Green Dao in the Android project, and set the repository and dependencies, configure the dependencies and configure the database related information in the Android file's build. gradle. The configuration database related information includes the database version number, the directory where the package name is located, and so on. After integration, GreenDao will automatically generate core management classes based on the configuration information.

\section{Function realization \\ GPS positioning function}

Visit the Gaode Map Open Platform, obtain the corresponding Gaode Key, and download the corresponding Gaode Map SDK to the Android project. Add Gaode Key in AndroidManifest.xml, add permissions to access mobile WiFi network information.Initialize the map container MapView in the layout file, create MainActivity, get the MapView control, manage the map life cycle, add the CircleProgressBar button of the third-party library in the user interface, and refresh the map for real-time positioning. 


\section{Borrowing umbrella function}

After the user logs in, the main interface displays Gaode map positioning, and the marker displayed on the map is the location of nearby umbrella borrowing points.Enter personal infoScan the code to unlock and pay. This function module introduces a third-party ZXing plug-in to obtain mobile phone camera permissions, integrates car information with a QR code scanning frame, reads it from the database, and displays it to the ListView control before borrowing the umbrella, save it to the database table MyInfo.

For teachers and students, shared umbrellas that can be borrowed on demand can avoid delays caused by sudden weather affecting travel and cause inconvenience to study and work. For resettlement sites with a large number of people, it can bring certain economic benefits to them (such as supermarkets) and achieve win-win cooperation; Because there is currently no mature umbrella service system on campus, this can also be a pioneering attempt for the school to improve the happiness of teachers and students.

\section{REFERENCE}

1. Eyu Chen, Yuwen Ren, Design and implementation of campus shared umbrella system based on internet of things [J], Journal of Jiujiang University (natural science edition). 2018,33(04):54-59

2. Huiying Tian. The design of shared product system under the concept of shared service [J], Journal of Weifang University. 2018,18(04):41-44

3. Jiafeng Ma, Zhipeng Wang, Design and realization of shared umbrella system [J], Electronic test. 2018(09):27-28

4. Boheng Xia. Design and realization of Shared umbrella system[D],Central China normal university; 2018

5. Yanchao Dong, Shared umbrella platform server design and key implementation[D], Zhejiang university; 2018

6. Shu Ya, Sun, Huan Fang, Optimization analysis of Petri net model of shared umbrella usage process[J],Journal of mudanjiang normal university (natural science edition); 2018(01):14-17

7. Gan Zhou, Jing Zhang, Based on Internet of things -- umbrella sharing system design [J], Computer knowledge and technology. 2017,13(21):99-101. 